\title{
TENTS guidelines: development of post-disaster psychosocial care guidelines through a Delphi process
}

Jonathan I. Bisson, Behrooz Tavakoly, Anke B. Witteveen, Dean Ajdukovic, Louis Jehel, Venke J. Johansen, Dag Nordanger, Francisco Orengo Garcia, Raija-Leena Punamaki, Ulrich Schnyder, A. Ufuk Sezgin, Lutz Wittmann and Miranda Olff

\section{Background}

How best to plan and provide psychosocial care following disasters remains keenly debated.

\section{Aims \\ To develop evidence-informed post-disaster psychosocial management guidelines.}

\section{Method}

A three-round web-based Delphi process was conducted. One hundred and six experts rated the importance of statements generated from existing evidence using a one to nine scale. Participants reassessed their original scores in the light of others' responses in the subsequent rounds.

\section{Results}

A total of 80 (72\%) of 111 statements achieved consensus for inclusion. The statement 'all responses should provide access to pharmacological assessment and management' did not achieve consensus. The final guidelines recommend that every area has a multi-agency psychosocial care planning group, that responses provide general support, access to social, physical and psychological support and that specific mental health interventions are only provided if indicated by a comprehensive assessment. Trauma-focused cognitivebehavioural therapy (CBT) is recommended for acute stress disorder or acute post-traumatic stress disorder, with other treatments with an evidence base for chronic post-traumatic stress disorder being made available if trauma-focused CBT is not tolerated.

\section{Conclusions}

The Delphi process allowed a consensus to be achieved in an area where there are limitations to the current evidence.

\section{Declaration of interest}

None.
There is increasing evidence concerning effective treatments of both chronic and acute trauma-related specific disorders, for example trauma-focused cognitive-behavioural therapy (CBT) for acute stress disorder or acute post-traumatic stress disorder (PTSD). ${ }^{1}$ On the contrary, high-quality research evidence is sparse in the area of post-disaster psychosocial management. The existing evidence is also not particularly helpful in determining exactly what should be done, especially very shortly after a traumatic event. ${ }^{2}$ It seems unlikely that we will be in a position to develop totally evidence-based guidelines in the foreseeable future. There is a need therefore to use the limited research evidence and expert opinion available to us in a meaningful way to develop guidelines on how best to provide psychosocial care. In order to address this issue the European Union funded a project, The European Network for Traumatic Stress (TENTS) (www.tentsproject.eu), to develop guidelines for psychosocial care following disasters that could be disseminated across Europe and, potentially, beyond Europe. Given the limited evidence base, it was decided to develop guidelines through achieving a consensus of expert opinion, guided by the evidence available, using a Delphi process.

\section{Method}

\section{Delphi process}

The Delphi process recognises the value of experts' opinions, experience and intuition when full scientific knowledge is lacking. ${ }^{3}$ It was developed, at the beginning of the cold war, as a systematic, interactive way for a panel of independent experts to forecast the impact of technology on warfare. ${ }^{4}$ A carefully selected group of experts answer surveys in two or more rounds. After each round, a facilitator provides an anonymous summary of the experts' views and their comments, allowing all participants to compare these with their own. The aim is that during this iterative process the range of the answers will decrease and the group will converge towards the 'correct' answer. ${ }^{5}$ Use of the Delphi process has resulted in widely accepted outcomes, including guidelines in the health field, without evidence of social conformism. ${ }^{6,7}$

\section{Statement development}

The results of systematic reviews of the evidence in this field along with existing guidelines and components widely cited as of potential benefit (e.g. specific formal interventions) following disasters were used to develop 96 statements. The statements covered six different domains of disaster management: planning, initial response (first week), early response (first month), response between 1 and 3 months, human resources and specific interventions. An example of a statement from each domain is shown in Appendix 1. A full list of the statements is available from the author on request. The online survey tool Survey Monkey (www.surveymonkey.com/) was used to present the statements with clear instructions to indicate level of agreement or disagreement using a nine-point scale where five was neutral. Participants were also asked to provide comments regarding their score for each statement. An example is given in Fig. 1.

In the second round, the survey comprised the same statements but this time with summary statistics indicating the number and percentage of participants who rated each score and the mean score for each statement. A summary of the comments was inserted underneath each statement for participants to consider when completing the second round. Some statements were 
Q-2. Every area should have a multi agency psychosocial care planning group.

$(1=$ Completely disagree; $9=$ Completely agree and $5=$ Neither $)$

\begin{tabular}{|l|l|l|l|l|l|l|l|l|l|}
\hline & 1 & 2 & 3 & 4 & 5 & 6 & 7 & 8 & 9 \\
\hline Rate: & & & & & & & & & \\
\hline
\end{tabular}

Comment:

Fig. 1 Example of a statement in round one.

amended slightly for clarification as a result of round one comments. An example is shown in Fig. 2. Participants were also sent a list of the full comments and their own scores. Fifteen new statements suggested by participants during round one were added to the end of the round two survey.

Any statements that had achieved a positive consensus (mean score $>7$ and $70 \%$ participants scoring 7 or above) were removed for round three along with any statements that were unlikely to achieve a positive consensus (those with a mean score of $<6$ ). Statements that scored between 6 and 7 were retained to determine whether positive consensus could be achieved. These statements were presented in the same format as the round two statements to illustrate the summary statistics and comments of the second round. The statements included for the first time in round two were retained for round three to allow participants the opportunity to reconsider their responses in the light of the comments of other participants and the summary statistics.

\section{Participants}

Potential participants were identified by the TENTS partners as having an expertise in the trauma field. They included researchers, clinicians, emergency planners, providers of psychosocial care following traumatic events and disaster survivors using services. The aim was to invite key individuals from countries throughout Europe and internationally recognised experts to participate in the survey.

\section{Analysis}

Basic statistics were generated by the Survey Monkey software. SPSS version 14 for Windows was used to determine which

Q-2<<<THERE IS GOOD CONSENSUS REGARDING THIS POINT >>>

Question amended to clarify: Every area (area size will vary according to country and organisation of services. Please consider an area to cover a population of roughly 250000 to 500000 people for the purpose of this survey) should have a multi-agency Psychosocial (addressing emotional, psychological and social needs) Care Planning Group.

$<1$ st Round Comments: Clarity requested regarding definition of 'area' and 'psycho-social'. Small areas might not have the resources. Should be on a national level. Key to the whole process.>

( 1 = Completely disagree; $9=$ Completely agree and $5=$ Neither $)$

\begin{tabular}{|l|c|c|c|c|c|c|c|c|c|}
\hline & 1 & 2 & 3 & 4 & 5 & 6 & 7 & 8 & 9 \\
& $(0.9 \%)$ & $(0.9 \%)$ & $(2.8 \%)$ & $(0.0 \%)$ & $(5.7 \%)$ & $(5.7 \%)$ & $(13.2 \%)$ & $(24.5 \%)$ & $(46.2 \%)$ \\
\hline $\begin{array}{l}\text { Rate (1st } \\
\text { round } \\
\begin{array}{l}\text { average } \\
\text { = 7.78): }\end{array}\end{array}$ & & & & & & & & & \\
\cline { 2 - 10 } & & & & & & & & & \\
\hline
\end{tabular}

Comment:

Fig. 2 Example of a statement in round two statements achieved positive consensus. The comments were summarised by J.I.B. to include the themes that emerged.

\section{Consultation}

Following completion of the three rounds of the Delphi process, the statements that achieved positive consensus were used to compile draft guidelines. These were circulated to the participants to comment on. The comments received were summarised and then discussed at a meeting of the TENTS partners to agree the final wording of the guidelines.

\section{Results}

\section{Round one}

One hundred and twenty-two potential participants were invited to take part in the first round. Of these, $106(87 \%)$ responded and $17(16.3 \%)$ identified themselves as researchers, $49(47.1 \%)$ as researcher-clinicians, $20(19.2 \%)$ as clinicians, $9(8.7 \%)$ as policy makers, $3(2.9 \%)$ as service users and $8(7.5 \%)$ as others. Online Table DS1 shows the number of respondents by country.

There did not appear to be significant differences in agreement with statements between respondents from different backgrounds or between respondents from different areas of the world. Fiftythree (55\%) of the original 96 statements achieved good consensus in round one and 15 new statements were formulated from respondents' suggestions. Twelve (12.5\%) statements were amended slightly to clarify their meaning as a result of the comments.

\section{Round two}

Round two was sent to the 106 round one respondents and 92 (87\%) completed it. Figure 3 shows the mean scores for rounds one and two showing an improvement in consensus for round two. Sixty-two $(65 \%)$ statements had achieved positive consensus, $8(8 \%)$ were in the 6-7 range and the remaining $26(27 \%)$ had a mean score of less than 6 . Six statements had a mean score of less than 3 and were considered to have achieved negative consensus. Thirteen $(87 \%)$ of the 15 new statements achieved positive consensus. The three statements that participants agreed most strongly with were: 'the psychosocial care plan should be incorporated into the overall disaster plan'; 'the initial response requires practical, pragmatic support provided in an empathic manner'; and 'community self-help and social support should be facilitated'. The three statements that participants disagreed most strongly with were: 'mental health professionals have no role in the initial response'; 'individuals should be discouraged from giving detailed accounts of their experiences'; 'all responses should provide formal single session early interventions such as psychological debriefing?.

\section{Round three}

Round three was sent to the 106 initial respondents. Ninety-two $(87 \%)$ completed it, $85(80 \%)$ completed all three rounds. Four $(50 \%)$ of the 8 original statements that were in the 6-7 range achieved positive consensus, $14(93 \%)$ of the 15 new questions did. This meant that a total of $80(72 \%)$ of the total 111 statements considered were deemed appropriate for inclusion in the guidelines. The included and excluded items are listed in the online supplement.

\section{Consultation}

The draft guidelines developed from the Delphi process comprised a brief introductory paragraph followed by six sections that 

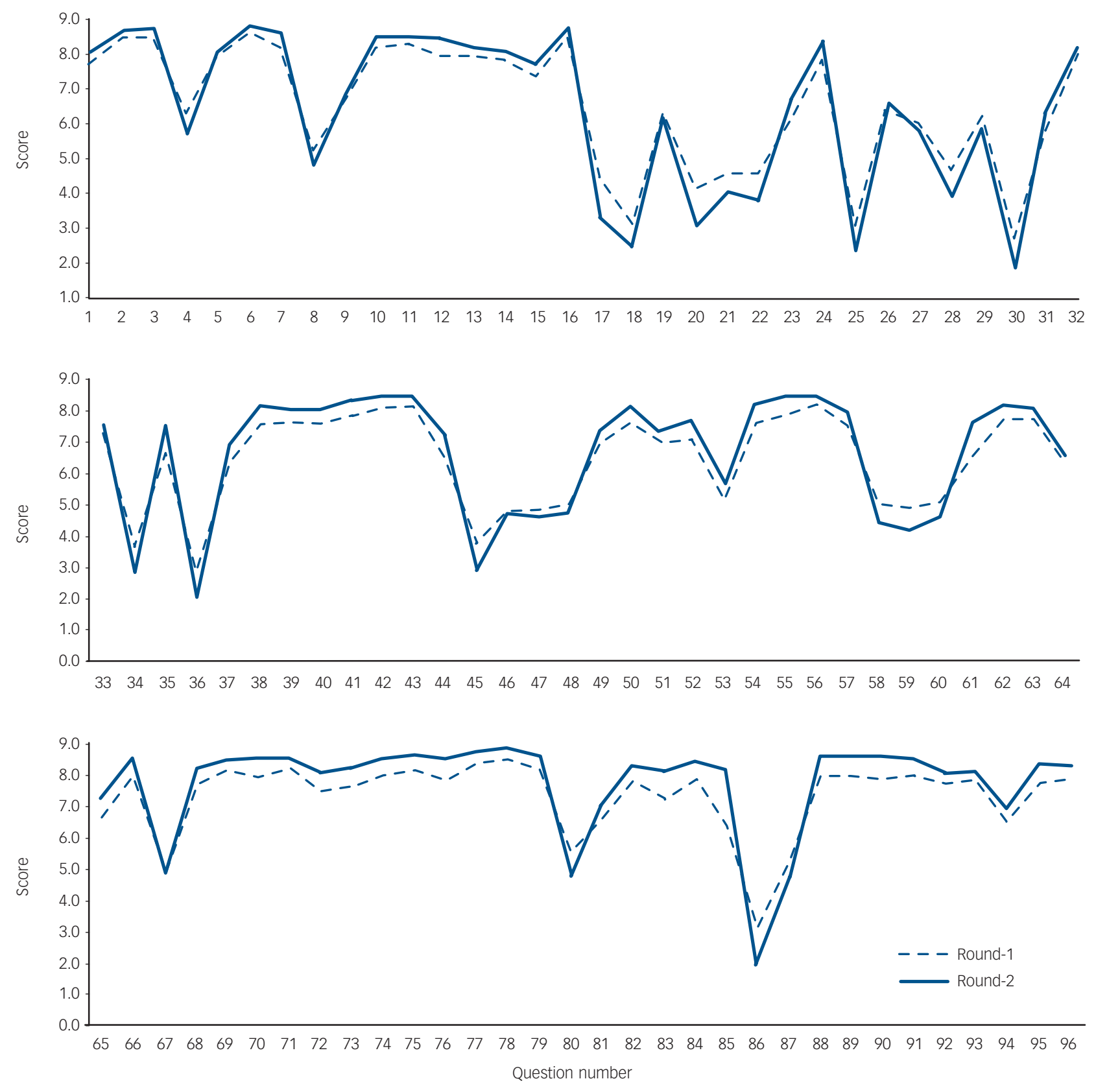

Fig. 3 Changes in mean scores for the 96 statements between rounds one and two.

covered: planning, preparation and management; general components; and specific components to be included within the first week, within the first month, 1 to 3 months after the disaster and beyond 3 months. Thirty-three (31\%) of the 106 round one respondents commented on the draft guidelines. The feedback received was very positive and included some suggested changes that primarily concerned the wording of the recommendations. The TENTS partners agreed that no statements should be altered in a way that contradicted what had been agreed during the Delphi process. Some minor changes to the wording of some statements were made. Some concerns were raised by partners that a number of the recommendations focused on individuals despite there being a strong consensus for a community-based approach. This was addressed by adding the following sentence to the introductory paragraph of the guidelines: 'Several of the components focus on individuals but all aspects of psychosocial care should only be provided with full consideration of individuals' wider social environment, especially their families and communities'. Appendix 2 lists the principal recommendations of the final guidelines, which are available in full on the TENTS website (www.tentsproject.eu).

\section{Discussion}

\section{Main findings}

The absence of high-quality research to inform the development of evidence-based guidelines is a major problem and can result in guidelines that are little more than the unchallenged views of a few people. The Delphi process allowed the development of a consensus of items that should be included in a psychosocial care guideline following disasters by an independent group of 106 experts from 25 different countries. The process allowed 
individuals to challenge the views of others in a non-threatening, anonymous manner, and it was apparent that many participants views changed to a degree as a result of the process. There were also statements where the consensus remained low suggesting that participants were not adjusting their responses to achieve conformity. The process allowed views to be aired regarding several issues that have been widely debated in the area, for example the use of single session psychological debriefing, producing some results that were not predictable, for example opposition to screening for everyone affected, as well as confirming a consensus that is largely consistent with what appears to be the prevailing view in this area.

\section{Early universal intervention}

The result of the Delphi process was a strong consensus against early application of formal intervention universally for all individuals involved in traumatic events. This concurs with findings of recent research ${ }^{8,9}$ and the considerable caution being expressed towards emerging interventions such as Psychological First Aid $^{10}$ and strong opposition to the inclusion of single session individual psychological debriefing. There is some evidence that debriefing can be associated with increased symptoms among trauma victims following some traumatic events. ${ }^{11,12}$

\section{Screening}

Screening those who might have been affected by a traumatic event for symptoms has been advocated despite studies that fail to support its implementation. ${ }^{13}$ Positive outcomes from a screening programme implemented after the July 2005 bombings in London have been reported although the researchers emphasised caution in drawing definitive conclusions regarding the effectiveness of screening programmes. ${ }^{14}$ The Delphi process resulted in consensus against formal screening of everyone affected. Participants commented that there was absence of evidence for effectiveness of screening, potential organisational difficulties and that scarce resources could be better allocated. Interestingly, the statement 'formal screening should not occur but helpers should be aware of the importance of identifying individuals with significant difficulties' achieved positive consensus in round two having not done so in round one.

\section{Pharmacological assessment and management}

The statement 'all responses should provide access to pharmacological assessment and management' did not achieve consensus after two rounds. The result is contrary to the widespread practice of prescribing medication to those who are affected by traumatic events. ${ }^{15}$ The comments about this statement suggested that participants were aware of the limited evidence base for pharmacological agents in the treatment of PTSD in the early response phases and their recommendation as a second-line therapy for PTSD. ${ }^{1}$ There is evidence for a small positive effect of medication for chronic PTSD and a better evidence base for other post-traumatic disorders such as depression.

\section{Psychoeducation}

The Delphi results showed a strong consensus for education being an important element in early management of those affected by disaster. Participants commented that by providing information about the consequences of trauma and education to the affected communities, the focus could be moved away from symptoms and towards resilience and natural human resources. This is against the findings of several randomised controlled trials that have failed to demonstrate a positive effect for education or providing self-help materials following traumatic events. ${ }^{18,19}$ Yet, the role of education in trauma and disaster is an area that has provoked a range of different views, and the Delphi process reflected that.

\section{Social support}

The participants demonstrated strong support for social approaches in helping those affected, which is consistent with findings that the presence of PTSD is associated with perceived lack of social support. ${ }^{18,19}$ However, no randomised controlled trials have addressed the role of social support in recovery from traumatic events. The Delphi process resulted in strong agreement with the five intervention principles proposed by Hobfoll and colleagues $^{2}$ that are supported by indirect evidence from various sources: promotion of sense of safety, sense of self- and community-efficacy, connectedness, calming and hope.

\section{Stepped care}

The guidelines are consistent with a stepped/stratified model of care, i.e. that routine formal psychological or pharmacological intervention for all affected individuals is not appropriate. Instead, individuals who develop symptoms may benefit from interventions, particularly trauma-focused CBT within the first 3 months for acute stress disorder and acute PTSD. ${ }^{1}$ The guidelines also deal with the very practical issue that not all symptomatic individuals benefit or can engage with trauma-focused CBT, by recommending the availability of alternatives with an evidence base for the treatment of chronic PTSD as second-line treatments (for example eye movement desensitisation and reprocessing and stress management). ${ }^{1}$

\section{Strengths and limitations}

A consensus approach to the development of guidelines should never replace a genuinely evidence-based approach or restrict efforts to achieve this. However, there is a requirement for psychosocial guidelines following disaster and we consider our approach the next best option, i.e. developing a consensus of rarely evidence-based, but usually evidence-informed, statements in a systematic manner. The Delphi process cannot develop high-level evidence but does avoid several potential problems encountered with non-systematic consensus development, for example failure to fully consider the limited evidence available, bias towards certain views and the dominance of certain individuals that often occurs in face-to-face group discussions. The high retention rate of participants across the three rounds was a major positive feature of this study, as was the care taken to systematically identify participants from a range of backgrounds and geographical areas as opposed to solely identifying individuals who belonged to a specific network or distinct professional background.

\section{Implications for practice}

The guidelines will evolve as our knowledge develops but represent the 2008 state of the art. They are much shorter than many recent documents and should be more accessible as a result. They are also written in a manner that is easy to translate into other languages. The results of the Delphi process strongly endorsed that such guidelines should not be mandatory. However, there was a consensus that planning in advance for disasters should occur along with training a workforce to respond appropriately to them. Such planning has long been in place for 
the non-psychosocial elements of responses to disaster and appears to result in good practice. The adoption of versions of these guidelines adapted for specific countries is likely to enhance preparedness for disasters and major incidents in the future.

Jonathan I. Bisson, DM, FRCPsych, Behrooz Tavakoly, PhD, Department of Psychological Medicine and Neurology, Cardiff University, University Hospital of Wales, Cardiff, UK; Anke B. Witteveen, PhD, Department of Psychiatry, Academic Medical Center, University of Amsterdam, The Netherlands; Dean Ajdukovic, PhD, Department of Psychology, Faculty of Humanities and Social Sciences, University of Zagreb, Zagreb, Croatia; Louis Jehel, MD, PhD, Department of Psychiatry, Hopital TENON, Paris, France Venke J. Johansen, PhD, Dag Nordanger, PhD, Nation ( Bergen, Norway; Francisco Orengo Garcia, MD, Sociedad Española de Bergen, Norway; Francisco Orengo Garcia, MD, Sociedad Española de Psicotraumatología, Estrés Traumático y Disociación, Madrid, Spain; Raija-Leena Punamaki, PhD, Docent Trauma Psychology, Helsinki Collegium of Advanced Studies, University of Helsinki, Finland; Ulrich Schnyder, MD, Department of Psychiatry, University Hospital Zurich, Switzerland; A. Ufuk Sezgin, PhD, Kocaeli University, Psychosocial Trauma Education, Research and Treatment Unit, Kocaeli, Turkey; Lutz wittmann, PhD, Department of Psychiatry, University Hospital Zurich, Switzerland; Miranda Olff, PhD, Center for Psychological Trauma, Psychiatry Academic Medical Centre, University of Amsterdam, Amsterdam, The Netherlands

Correspondence: Jonathan I. Bisson, Cardiff University, Monmouth House, University Hospital of Wales, Heath Park, Cardiff, CF14 4XN, UK. Email: bissonji@cf.ac.uk

First received 20 Mar 2009, final revision 7 Aug 2009, accepted 21 Aug 2009

\section{Funding}

European Union.

\section{Acknowledgements}

We wish to express our thanks to all participants in the Delphi process

\section{Appendix 1}

\section{Delphi process statement examples}

$\begin{array}{ll}\text { Domain } & \text { Statement } \\ \text { Planning } & \text { A European plan should be made mandatory for all } \\ & \text { countries }\end{array}$

Initial response The initial response should promote connectedness (first week)

Early response All individuals should be screened for mental health (first month) difficulties using a structured questionnaire or interview

Response Individuals with difficulties should be formally assessed between 1 and 3 with consideration for their physical, psychological and months social needs before receiving any specific intervention

Human resources All responders should have undergone formal training

Specific services All responses should provide formal single session early interventions such as psychological debriefing

\section{Appendix 2}

\section{Principal recommendations of the TENTS guidelines}

Multi-agency planning group: every area should have a multi-agency psychosocial care planning group that includes mental health professionals with expertise in traumatic stress who have a designated responsibility for psychosocial care following disasters and major incidents. Individuals affected by disasters or major incidents should also be represented.

Psychosocial care plan: every area should have guidelines on the provision of psychosocial care in emergencies (a psychosocial care plan) that are incorporated into the overall disaster/major incident plan and regularly updated.
Training and supervision: all care providers should have undergone formal training and receive ongoing training, support and supervision.

Response: the response should promote a sense of safety, self and community efficacy/empowerment, connectedness, calm and hope.

Responses should provide general support, access to social support, physical support and psychological support.

Formal early interventions for all: provision of specific formal interventions such as single session individual psychological debriefing for everyone affected should not occur.

Social support: efforts should be made to identify the correct supportive resources (e.g. family, community, school, friends, etc).

One-stop shop: a humanitarian assistance centre/one-stop shop should be established where a range of services potentially required can be based.

Symptomatic individuals: individuals with psychosocial difficulties should be formally assessed by a trained professional with consideration for their physical, psychological and social needs before receiving any specific intervention.

Trauma-focused cognitive-behavioural therapy (CBT): treatment with trauma-focused CBT should be available for individuals with acute stress disorder or acute post-traumatic stress disorder (PTSD).

Other evidence-based treatments: other treatments with an evidence base for chronic PTSD should be available for individuals with acute PTSD when trauma-focused CBT is not available or is not tolerated.

Evidence-based interventions for individuals with other mental health difficulties should be available.

Long-term coordinated planning and cooperation: detailed planning should occur with local authorities/governments and existing services to fund and provide appropriate extra provision to support local services for several years following the disaster.

\section{References}

1 National Collaborating Centre for Mental Health. Post-traumatic Stress Disorder (PTSD) The Management of PTSD in Adults and Children in Primary and Secondary Care. (CG 26). National Institute for Health and Clinical Excellence, 2005.

2 Hobfoll SE, Watson P, Bell CC, Bryant RA, Brymer MJ, Friedman MJ, et al. Five essential elements of immediate and mid-term mass trauma intervention: empirical evidence. Psychiatry 2007; 70: 283-315.

3 Linstone HA, Turoff M. The Delphi Method: Techniques and Applications. Addison-Wesley, 1975

4 Dalkey NC, Helmer O. An experimental application of the Delphi method to the use of experts. Manag Sci 1963; 9: 458-68.

5 Skulmoski GJ, Hartman FT, Krahn J. The Delphi method for graduate research. J Inf Tech Educ 2007; 6: 1-21.

6 Langlands RL, Jorm AF, Kelly $\mathrm{CM}$, Kitchener BA. First aid recommendations for psychosis. Using the Delphi method to gain consensus between mental health consumers, carers, and clinicians. Schizophr Bull 2008; 34: 435-43.

7 Campbell SM, Cantrill JA, Roberts D. Prescribing indicators for UK general practice: Delphi consultation study. BMJ 2000; 321: 425-8.

8 Rose S, Bisson Jl, Churchill R, Wessely S. A systematic review of brief psychological interventions ("'debriefing") for the treatment of immediate trauma related symptoms and the prevention of posttraumatic stress disorder. Cochrane Database Syst Rev 2005; 2: CD000560.

9 Roberts NP, Kitchiner N, Kenardy J, Bisson J. Systematic review and metaanalysis of multiple session early interventions following traumatic events. Am J Psychiatry 2009; 166: 293-301.

10 National Child Traumatic Stress Network and National Center for PTSD. Psychological First Aid: Field Operations Guide (2nd edn). NCTSN \& NCPTSD, 2006. 
11 Bisson Jl, Jenkins PL, Alexander J, Bannister C. Randomised controlled trial of psychological debriefing for victims of acute burn trauma. Br J Psychiatry 1997; 171: 78-81.

12 Hobbs M, Mayou R, Harrison B, Worlock P. A randomised controlled trial of psychological debriefing for victims of road traffic accidents. BJM 1996; 313 1438-9.

13 Rona RJ, Jones M, French C, Hooper R, Wessely S. Screening for physical and psychological illness in the British Armed Forces: the acceptability of the programme. J Med Screen 2004; 11: 148-52.

14 Brewin CR, Scragg $P$, Robertson $M$, Thompson $M$, d'Ardenne $P$, Ehlers $A$, et al. Promoting mental health following the London bombings: a screen and treat approach. J Traum Stress 2008; 21: 3-8.

15 Mellman T, Clark R, Peacock W. Prescribing patterns for patients with posttraumatic stress disorder. Psychiatr Serv 2003; 54: 1618-21.
16 Rose S, Brewin CR, Andrews A, Kirk M. A randomized controlled trial of psychological debriefing for victims of violent crime. Psychol Med 1999; 29 793-9.

17 Scholes C, Turpin G, Mason S. A randomised controlled trial to assess the effectiveness of providing self-help information to people with symptoms of acute stress disorder following a traumatic injury. Behav Res Ther 2007; 45 2527-36.

18 Brewin CR, Andrews B, Valentine JD. Meta-analysis of risk factors for posttraumatic stress disorder in trauma-exposed adults. J Consult Clin Psychol 2000; 68: 748-66.

19 Ozer EJ, Best SR, Lipsey TL, Weiss DS. Predictors of post-traumatic stress disorder and symptoms in adults: a meta-analysis. Psychol Bull 2003; 129 $52-73$.

Af EXTRA

\section{Poems \\ by doctors}

\section{A Lover's Quarrel}

\section{Vincent Hanlon}

Describe it simply as a lovers' quarrel after which she slashed her wrist and sought medical attention.

\section{A superficial laceration}

over the right wrist.

No active bleeding.

Clean the area with antiseptic

and pick away the dried clot.

Tidy tidy.

A scratch on the skin

parallels the main cut:

presumably an initial

tentative testing of the flesh.

Don't look here

for the confident opening incision

of a surgeon amputating a limb.

Instead, observe the hesitant and unsuccessful

pruning of the hand that caresses.

What is a doctor to do?

So late at night.

First of all, be gentle with the tissues.

Restore the natural anatomy

With a close approximation of the skin edges.

Use a small needle.

Minimize the small talk.

'You are very brave,'

she says when we're finished.

I don't argue.

It's too early in the morning

to start another lovers' quarrel.

Vincent Hanlon studied English literature and later medicine at University of Calgary. He is an emergency physician at Fort McMurray, Alberta. This poem is from The Naked Physician: Poems about the Lives of Patients and Doctors, edited by R. Charach (Quarry Press). Reprinted with kind permission of the author.

Chosen by Femi Oyebode. 